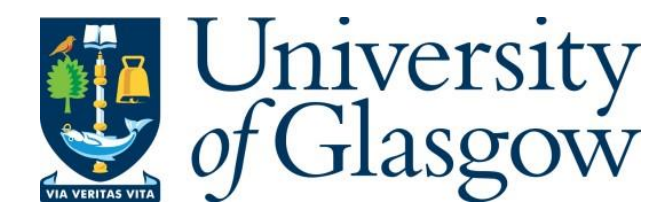

Yang, K., Abbasi, Q. H., Fioranelli, F., Romain, O. and Le Kernec, J. (2022) Bespoke Simulator for Human Activity Classification with Bistatic Radar. In: 16th EAI International Conference on Body Area Networks (EAI BODYNETS 2021), Glasgow, UK, 25-26 Oct 2021, pp. 71-85. ISBN 9783030955922.

There may be differences between this version and the published version. You are advised to consult the publisher's version if you wish to cite from it.

https://eprints.gla.ac.uk/250636/

Deposited on: 31 August 2021

Enlighten - Research publications by members of the University of Glasgow https://eprints.gla.ac.uk 


\title{
Bespoke simulator for human activity classification with bistatic radar
}

\author{
Kai Yang ${ }^{1,2}$, Qammer H. Abbasi ${ }^{1[0000-0002-7097-9969]}$, Francesco Fioranelli ${ }^{3[0000-0001-8254-}$ \\ ${ }^{8093]}$, Olivier Romain ${ }^{40000-0002-2172-1865]}$ and Julien Le Kernec ${ }^{1,2[0000-0003-2124-6803]}$ \\ ${ }^{1}$ University of Glasgow, University Avenue, G12 8QQ, Glasgow, UK. \\ ${ }^{2}$ University of Electronic Science and Technology of China, Qingshuihe Campus: Chengdu \\ High-tech Zone (West) 2006 West Avenue, source Zip: 611731, China. \\ ${ }^{3}$ TU Delft, Mekelweg 4, 2628 CD Delft, The Netherlands. \\ ${ }^{4}$ University Cergy-Pontoise, 6 av du ponceau, 95000 Cergy-Pontoise, France. \\ julien.lekernec@glasgow.ac.uk
}

\begin{abstract}
Radar is now widely used in human activity classification because of its contactless sensing capabilities, robustness to light conditions and privacy preservation compared to plain optical images. It has great value in elderly care, monitoring accidental falls and abnormal behaviours. Monostatic radar suffers from degradation in performance with varying aspect angles with respect to the target. Bistatic radar may offer a solution to this problem but finding the right geometry can be quite resource-intensive. We propose a bespoke simulation framework to test the radar geometry for human activity recognition. First, the analysis focuses on the monostatic radar model based on the Doppler effect in radar. We analyse the spectrogram of different motions by Short-time Fourier analysis (STFT), and then the classification data set was built for feature extraction and classification. The results show that the monostatic radar system has the highest accuracy, up to $98.17 \%$. So, a bistatic radar model with separate transmitter and receiver was established in the experiment, and results show that bistatic radar with specific geometry configuration (CB2.5) not only has higher classification accuracy than monostatic radar in each aspect angle but also can recognise the object in a wider angle range. After training and fusing the data of all angles, it is found that the accuracy, sensitivity, and specificities of CB2.5 have $2.2 \%, 7.7 \%$ and $1.5 \%$ improvement compared with monostatic radar.
\end{abstract}

Keywords: Radar, Micro-Doppler, Radar Signature Simulation, Human Activity Recognition.

\section{Introduction}

Human activity recognition has become one of the current research hotspots. It can help to monitor different human behaviour states. Therefore, this technology has been widely used in security monitoring and medical health [1-3]. Nowadays, the most commonly used sensor in recognition is a camera, which can extract features by recording images of human motions, and then perform classification through machine learning or 
deep learning algorithms. However, the method of camera recording also has some drawbacks [4-7]. Images collected by the camera are easily affected by light conditions in the environment, and in addition, camera recording may infringe on personal privacy and may cause information leakage [6].

Because the use of a camera in private spaces is raising concerns with privacy and acceptance by users, radar is becoming an attractive sensing modality $[8,9]$. Radar can see through walls and maintain good performance in any lighting conditions. Radar can penetrate obstacles to detect hidden targets. Finally, radar is a non-contact device. These benefits mean that radar technology has great application value in human activity recognition.

The world's ageing problem is getting worse, e.g., the proportion of the world's elderly population reached $9 \%$ in 2019 . China is also facing a serious ageing problem. China Development Report [10] predicts that China's population over 65 will account for $14 \%$ in 2022, and the population over 60 years old will reach 500 million by 2015 . Therefore, how to ensure the quality of life of the elderly and establish a complete medical and elderly care system will be a great challenge in China and in the world. In the daily life of the elderly, there are often unexpected falls or abnormal behaviours caused by diseases, which can be life-threatening in the case of unattended [11]. Thus, it is necessary to monitor Activities of Daily Living (ADL) of the elderly through equipment.

There have been much research works on the use of radar for human activity recognition. Chen proposed to use the micro-Doppler effect $[12,13]$ to obtain information on human motions. By using joint time-frequency analysis of radar signals, a time-dependent spectrum (spectrogram) description can be obtained. The motion of different body parts on the spectrum has different Doppler frequency shift trajectories. Therefore different motions will have different micro-Doppler signatures. There are short-time Fourier transform (STFT), continuous wavelet transform (CWT), Wigner Ville distribution (WVD) [14] for time-frequency transformation, while STFT is the most commonly used technology. Spectrogram data will be further processed to extract features. One method is physical component-based decomposition [15], which decomposes the spectrogram into components corresponding to different physical parts of the human body. However, how to track and decompose these signatures effectively is still complex, so it is challenging to implement this method. Another method is statistics-based decomposition, which is a feature decomposition method using Principal Component Analysis (PCA) or Singular Value Decomposition (SVD) [16,17]. Through this method, the information with a small correlation is removed, and the main information is retained, which has the effect of data dimension reduction and noise reduction. In [18], PCA is used to extract the feature of the spectrogram. For classification, machine learning $[16,17]$ is a powerful tool to automate the task with algorithms such as K-Nearest Neighbor (KNN) is used in [19], Decision Tree and Naive Bayesian are used in [20], their final classification accuracy is greater than $90 \%$. Most of the research on radar recognition for human activities are based on monostatic radar. However, the transmitter and receiver of monostatic radar are in the same position, so the observation angle is limited, and significant Doppler shift for classification purposes can be obtained only when the subject moves towards or away from the radar (when the aspect angle of the 
subject to the radar line of sight is $0^{\circ}$ or $180^{\circ}$ ). The Doppler shift will be greatly reduced when the aspect angle is $90^{\circ}$, resulting in a significant decline in the classification performance of the spectrogram [21].

The transmitter and receiver of bistatic radar are separated and located in different positions, which can offer more freedom of acquiring complementary target's information, avoiding aspect angles with poor velocity measurements. Recording bistatic information is, however, demanding in terms of hardware resources to synchronise the radar nodes [22]. Researchers, therefore, turn to bespoke simulations to generate radar signatures that can complement experimental radar data [23-28]. The problem of aspect angle in [21,29-31] and its effect on radar activity classification is seldom analysed in its entirety, as many studies consider target classification with subjects mostly moving in the radial direction in constrained trajectories. A person cannot be expected to always be facing a radar node. This would be impractical in daily life.

Because the aspect angle of the target influences the radar signatures, this has an influence on the micro-Doppler signatures and, therefore, the classification accuracy. Exploiting different radar geometry may help to counter the degradation with aspect angle. This paper proposes a parametric analysis of bistatic radar geometries to maintain performance with varying aspect angles from $-90^{\circ}$ to $90^{\circ}$ using a bespoke simulation framework. It first analyses the suitability of the classification algorithm for practical implementation using the monostatic model. Secondly, the classification performances are tested when one algorithm is trained for signatures produced at each aspect as well as when one model is trained with the data from all angles.

The remainder of this paper is organised as follows. Section 2 will present the simulation framework. Section 3 will discuss the classification methods. Section 4 will provide the result of the classification of human activities. Section 5 will offer some insight in the results obtained from classification. Finally, Section 6 will conclude and provide future research directions.

\section{Radar Simulation}

The simulated radar operating frequency was set to $15 \mathrm{GHz}$, the range resolution is 0.01 $\mathrm{m}$, and the monostatic radar model is based on [12], which uses canonical shapes to emulate the radar cross-section (RCS) of the human body, such as spheres and ellipsoids. Whereas in [12] the Boulic-Thalmann model [32] was used, this paper combines human motion capture (MOCAP HDM05) data containing the motion information of the human skeleton in each frame, sampled at $120 \mathrm{~Hz}$ [33]. This allows for a wider variety of actions to be explored for classification as the MOCAP dataset is not limited to an average subject and walking gait, as the Boulic-Thalmann model.

In addition to monostatic radar, this paper also explores bistatic configurations, for which the bistatic RCS of spheres and ellipsoids can be found in [34,35]. The transmitter and receiver of bistatic radar are located in different positions, and there can be multiple transmitters and receivers. This allows the bistatic radar to have more observations, which may improve classification with varying target aspect angles. However, bistatic radar also leads in practical implementations to a more complex system and to 
the synchronisation challenge between transmitter and receiver. Hence, the value of establishing a simulator to get some initial data.

The baseline between transmitter and receiver is $\mathrm{L}, r_{T}$ and $r_{R}$ is the distance from the transmitter to target and receiver to target, respectively, $\alpha_{T}$ and $\beta_{T}$ is the azimuth and elevation angle of the transmitter, $\alpha_{R}$ and $\beta_{R}$ is the azimuth and elevation angle of the receiver. The angle between the transmitter-to-target line and the receiver-to-target line is the bistatic angle $\varphi$ (the angle between the moving direction of the target and the direction of the bisector).

There are three main factors that affect the Doppler shift in the bistatic radar system: the velocity of target $\mathrm{V}$, the angle $\varphi$ and the angle $\delta$, so the formula of Doppler frequency shift is shown in (1).

$$
f_{D}=\frac{2 f}{c}|\boldsymbol{V}| \cos \left(\frac{\varphi}{2}\right) \cos \delta(1)
$$

The received signal can be expressed as (2).

$$
S(t)=\rho(x, y, z) \exp \left\{\left\{2 \pi f \frac{\left|\mathbf{r}_{T}(t)\right|+\left|\mathbf{r}_{R}(t)\right|}{c}\right\}(2)\right.
$$

Note that the sampling frequency of the HDM05 database is only $120 \mathrm{~Hz}$, and the Doppler frequency shift generated by human motion is often larger. This will cause the aliasing of the spectrogram according to Shannon-Nyquist sampling theory. Therefore, the HDM05 data is interpolated to increase the sampling frequency to $2 \mathrm{kHz}$, which ensures that the spectrogram has $\mathrm{a} \pm 1 \mathrm{kHz}$ Doppler unambiguous range, which is sufficient for the activities being considered. The spectrogram is generated using a 150point Gaussian window with 95\% overlap and 600-point FFT.

Five motion classes are considered: Walk, Jumping Jack, Hop, Squat, and Rotate Arms with associated labels from 1 to 5 , in the same order. The dataset contains 130 samples from the HDM05 database. To obtain more data for machine learning, it is necessary to expand the dataset. The spectrogram is therefore segmented in smaller chunks, and a sliding window will be used. The window size is set to be $1.5 \mathrm{~s}$ to make sure at least one complete cycle of walking can be covered. Besides, there is a $33 \%$ overlap between each window. After segmentation, 71 samples were obtained for each motion, a total of 355 samples, and the size of each spectrogram is $600 \times 471$. In addition, for a more realistic simulation, additive white Gaussian noise (AWGN) was added to the samples to develop robustness against varying signal-to-noise ratio (SNR) levels $(-5 \mathrm{~dB}, 0 \mathrm{~dB}, 5 \mathrm{~dB}$ and $10 \mathrm{~dB})$ in the classification algorithms. To further increase the dataset, flipping the radar signatures upside down to obtain Doppler for a motion performed in the opposite direction of the original one allows doubling the size of the dataset. Finally, there are a total of 2840 samples (568 per class). The radar signatures obtained with a simulated monostatic radar configuration are shown in Fig. 1. All the simulations were performed in Matlab. 


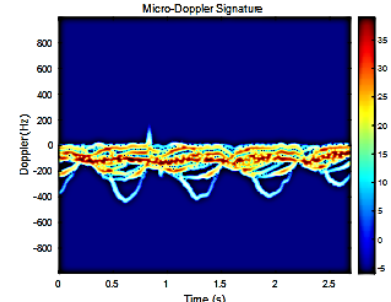

(a)

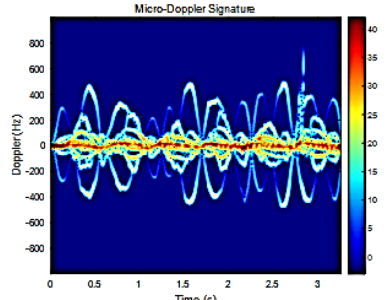

(b)

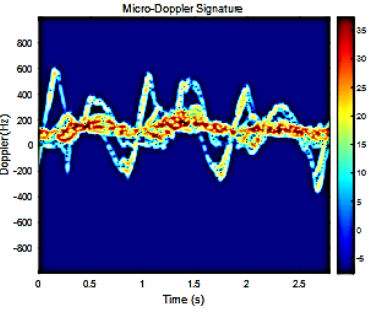

(c)

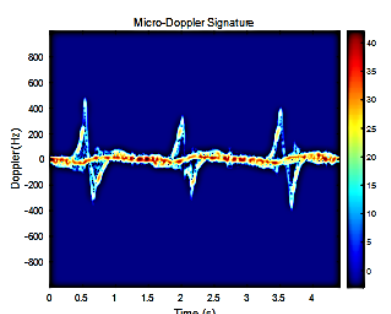

(d)

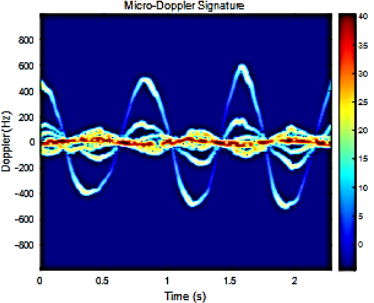

(e)

Fig. 1. Spectrograms of (a)Walking (b) Jump jack (c) Hop (d) Squat (e) Rotate arms in a monostatic radar environment.

In bistatic radar, the receiver, transmitter, and target are set, as shown in Fig. 2. Different geometries lead to different aspect angles, which alter the micro-Doppler signatures. Three circular bistatic radar (CB) geometries are considered with baselines of 10 $\mathrm{m}(\mathrm{CB} 10), 5 \mathrm{~m}$ (CB5) and $2.5 \mathrm{~m}$ (CB2.5), respectively. The distance to the centre of the scene is maintained at $7 \mathrm{~m}$. The aspect angle $\theta$ is defined as the angle between the target heading and the radar line of sight when considering the transmitter. The effect of varying aspect angles (- $90^{\circ}$ to $90^{\circ}$ with $15^{\circ}$ steps) for different geometries for the walking activity is shown in Fig. 3.

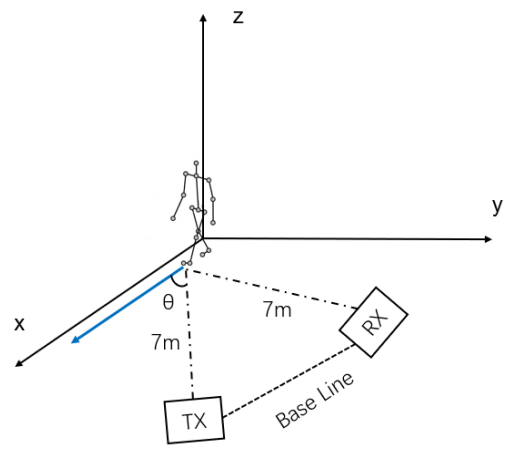

Fig. 2. The simulation geometry of a bistatic radar 

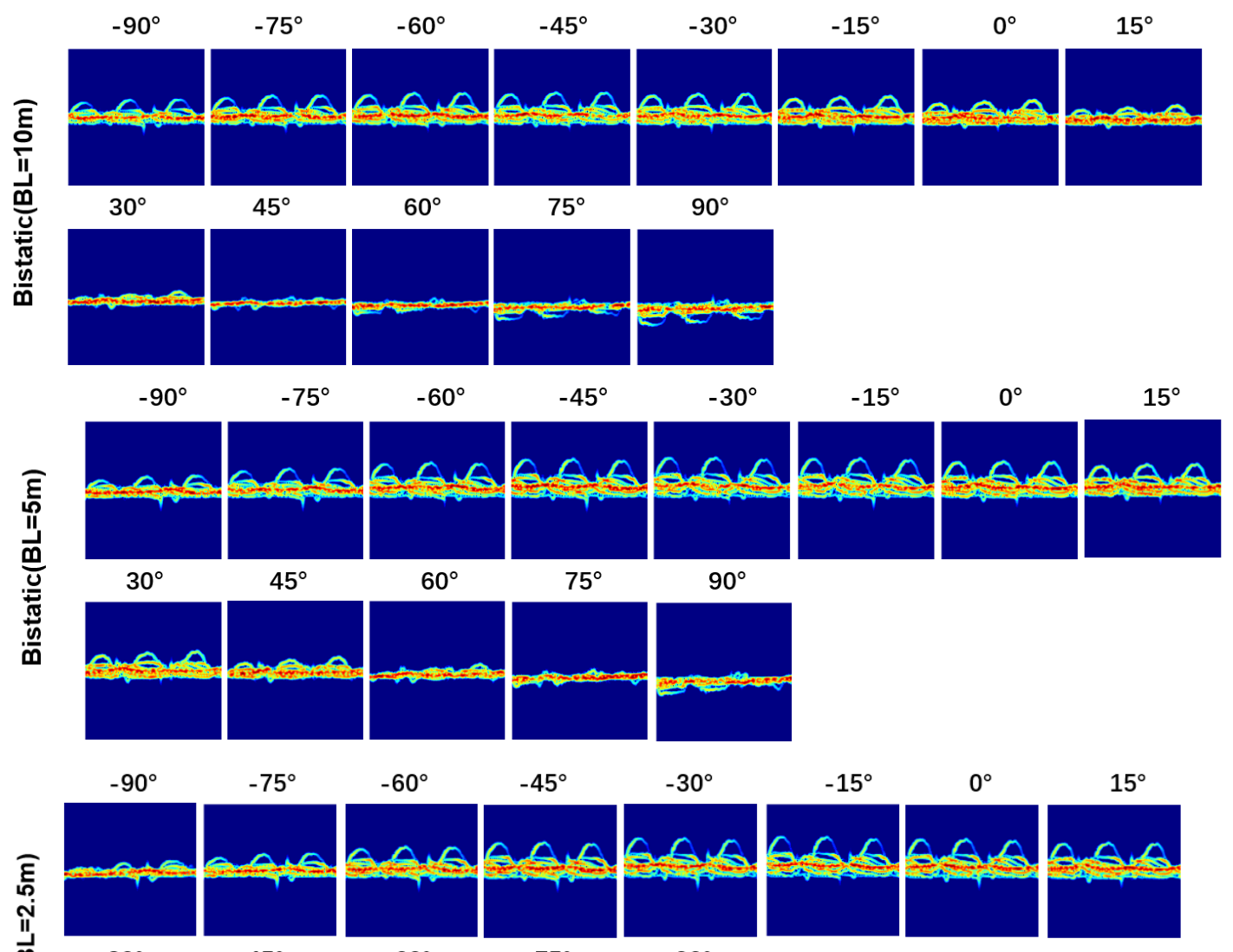

$\begin{array}{lllll}30^{\circ} & 45^{\circ} & 60^{\circ} & 75^{\circ} & 90^{\circ}\end{array}$
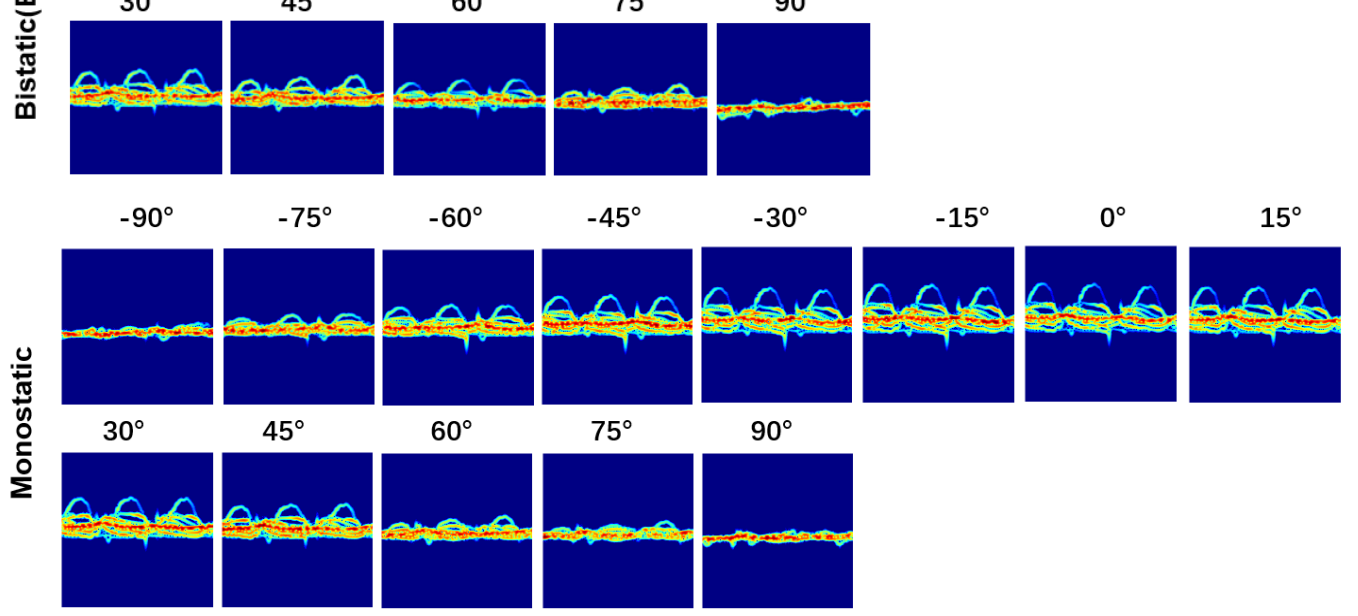

Fig. 3. Spectrograms for walking gait for configuration CB10, CB5, CB2.5 as bistatic geometries and for the monostatic radar case, when aspect angle changes from $90^{\circ}$ to $90^{\circ}$ 


\section{Classification}

\subsection{Feature Extraction}

The spectrogram data can be used directly for classification. However, this increases computational complexity if the spectrogram is used as an image directly. Therefore, it is recommended to reduce the dimensionality of the data and extract the salient information for the given classification task. The traditional PCA will transform the spectrogram data into a one-dimensional vector, therefore, losing the spatial information. Hence, 2D-PCA [36] is used to extract features from the spectrograms. Compared with traditional PCA, 2D-PCA can evaluate the covariance matrix more accurately and compute the eigenvalues faster than PCA by at least 4 times but requires more components to describe the images. The comparison of reconstruction images for the varying numbers $d$ of PCA is shown in Fig. 4. The quality of the image improves as $d$ increases visually. However, through the KNN classifier (with the number of neighbours $\mathrm{K}=5$ ), it is found that the highest accuracy is obtained when $d=15$, as shown in Fig. 5. Furthermore, a higher $d$ value will result not just in a lower accuracy but also an increase in computational requirements resulting in a longer inference time.

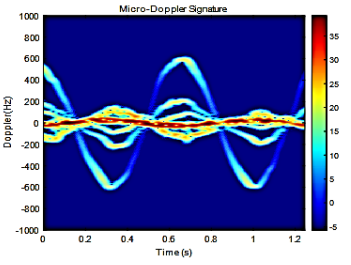

(a)

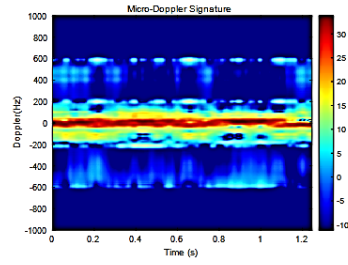

(b)

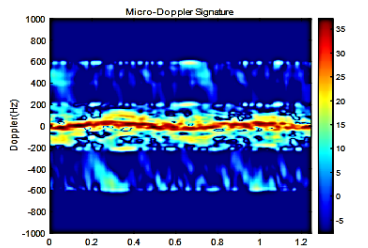

(c)

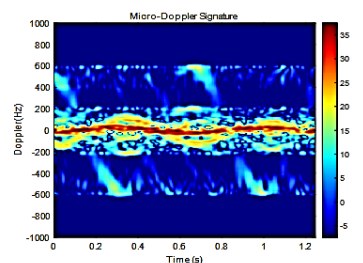

(d)

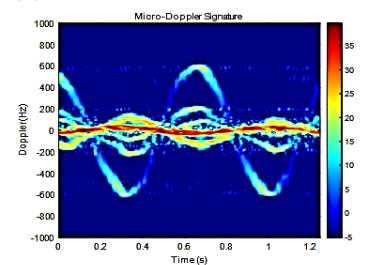

(e)

Fig. 4. (a) Original spectrogram (b) Reconstructed spectrogram $(d=5)$ (c) Reconstructed spectrogram $(d=15)(\mathrm{d})$ Reconstructed spectrogram $(d=25)$ (e) Reconstructed spectrogram $(d=50)$

\subsection{Classification algorithm}

Four classification algorithms are evaluated in this study, namely, KNN, Support Vector Machine (SVM) with a linear kernel, Decision Tree, and Random Forest (RF) [17]. These four algorithms have different advantages and disadvantages, so it is necessary to evaluate the classification effectiveness via various metrics, such as accuracy, specificity, sensitivity, training time and inference time.

To verify the classification performance, 10 -fold cross-validation will be used. The data set is divided into ten parts: one is the test set and the rest the training set; this 
process is then repeated ten times until all parts are used as the test set. The following section presents the average results of the 10 folds.

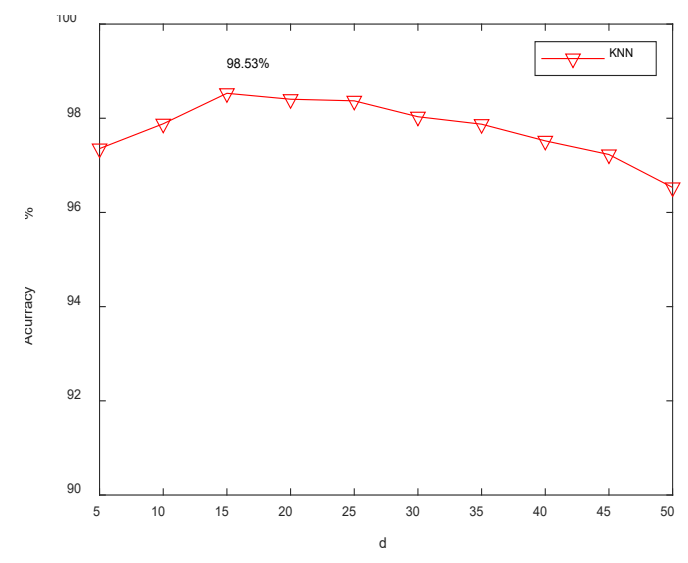

Fig. 5. Accuracy of classification of the 5 activities with $\mathrm{KNN}(\mathrm{K}=5)$ against the number of PCA values $d$

\section{$4 \quad$ Classification results}

\subsection{Monostatic results}

Some results are presented regarding performances with monostatic configuration. Confusion matrices of the different models are shown in Fig. 6. The average accuracy, sensitivity and specificity are shown in Table 1 . The stability of the algorithms is shown in Fig. 7 with boxplots. Finally, the evaluation of classification efficiency with training time and inference time is shown in Fig. 8. From these results, RF achieves the highest accuracy (98.17\%), sensitivity $(95.42 \%)$ and specificity $(98.86 \%)$; the decision tree has the worst classification performance. Furthermore, Fig. 7 shows that RF has a smaller deviation overall indicating better stability in performance, and KNN has the worst deviation. For the comparison of training time, SVM takes the longest time, while in inference time, it is faster than RF and KNN. Since training happens off-line, faster inference time and robust accuracy will be favoured for practical implementation. Hence, SVM is retained for further analysis.

\subsection{Bistatic results}

To verify the classification performance of radars under different aspect angles $\left(-90^{\circ}\right.$ to $\left.90^{\circ}\right)$, datasets are constructed for each rotation for the different radar geometries (monostatic, CB10, CB5, CB2.5). The accuracy for each aspect angle is shown in Fig. 9. Furthermore, the data from all the aspect angles are fused together to train a general model for each radar geometry, and the results are shown in Fig. 10. 
The overall performance of CB2.5 is better since its accuracy in the range of - $75^{\circ}$ to $75^{\circ}$ is higher than that of the other radar geometries. Furthermore, the fusion data model in Fig. 10. shows that CB2.5 has higher accuracy, sensitivity and specificity than the other configurations as well.

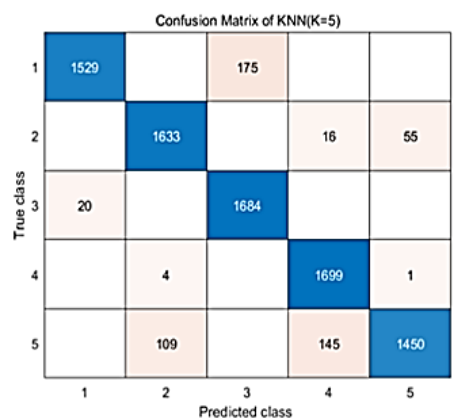

(a)

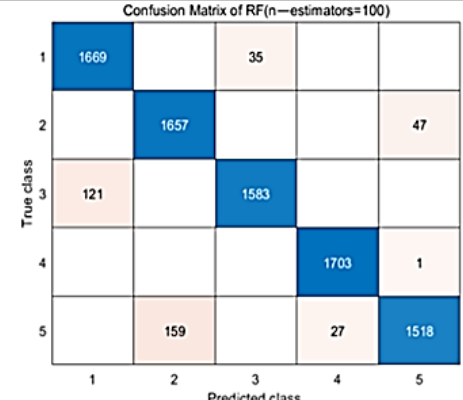

(c)

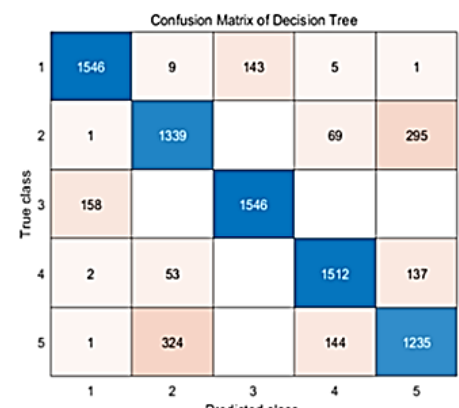

(b)

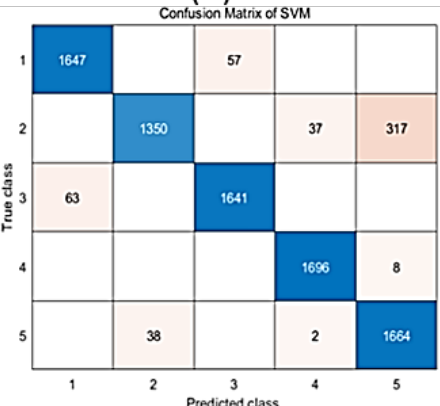

(d)

Fig. 6. Confusion Matrices of (a) KNN (b) Decision Tree (c) Random Forest (d) SVM

Table 1. Average accuracy, sensitivity, specificity of 4 classifiers in monostatic radar system

\begin{tabular}{l|llll} 
Classifier & KNN & Decision Tree & RF & SVM \\
\hline Accuracy & 0.9754 & 0.9370 & 0.9817 & 0.9755 \\
Sensitivity & 0.9384 & 0.8425 & 0.9542 & 0.9387 \\
Specificity & 0.9846 & 0.9606 & 0.9886 & 0.9847
\end{tabular}




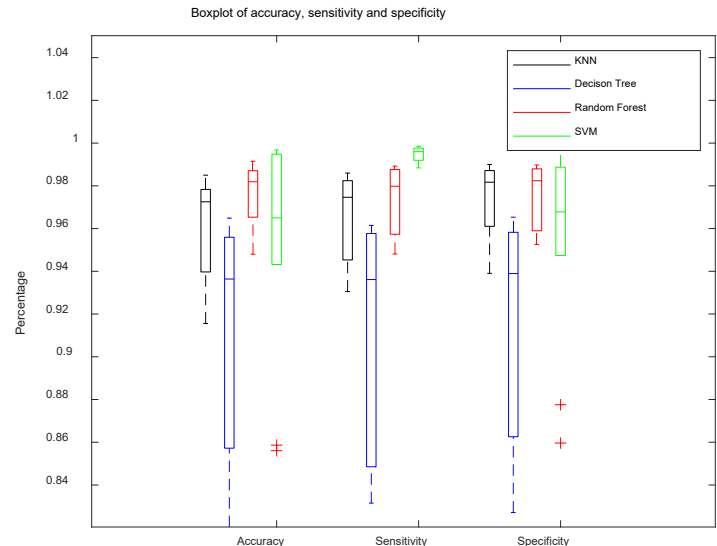

Fig. 7. Boxplot of accuracy, sensitivity and specificity in 10-fold cross-validation.
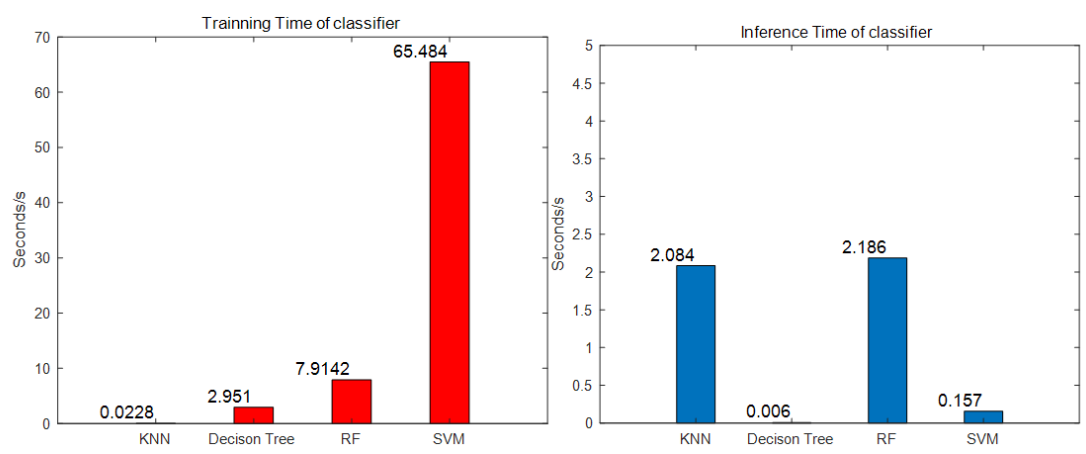

Fig. 8. Average (left) training time (right) inference time of 10 -fold cross-validation.

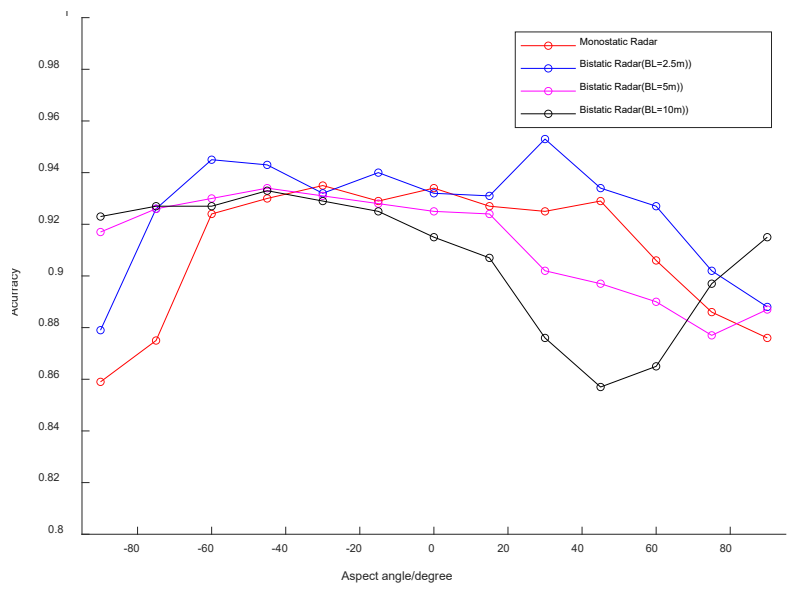

Fig. 9. Accuracy of CB10, CB5, CB2.5 and Monostatic Radar under different aspect angles with SVM. 


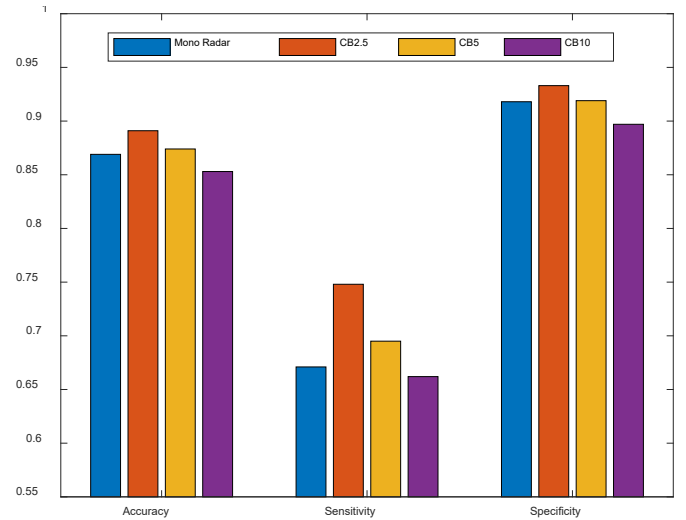

Fig. 10. Comparison of accuracy of different radar geometries after data fusion of all aspect angles with SVM

\section{Discussion}

\subsection{Monostatic}

We first completed the motion classification with a monostatic radar. Four machine learning algorithms are applied to compare which one will have better classification performance on the dataset. The classification results in Table 1 show that, except for the Decision Tree, the other three classifiers all achieve a good classification performance, their average accuracy all exceed 90\%, and RF has the best accuracy (98.17\%), sensitivity (95.42\%) and specificity $(98.86 \%)$, followed by SVM and KNN, while Decision Tree has the worst classification performance, its sensitivity is about $10 \%$ lower than the other three classifiers.

In Fig. 7, the width of the box reflects the fluctuation of the data. It illustrates that RF has the smallest fluctuations in accuracy, while SVM has a more obvious advantage in sensitivity. In specificity, KNN is slightly better than RF. RF is a relatively stable model in all three performance parameters. On the contrary, Decision tree shows the poorest performance in accuracy, sensitivity and specificity, so the stability of the model is the worst. In the comparison of time spent, Fig. 8 shows that the training time of SVM $(65.48 \mathrm{~s})$ is much longer than the others, followed by RF (7.91 s) and Decision Tree $(2.95 \mathrm{~s}$ ), KNN has the shortest training time (only $0.0228 \mathrm{~s}$ ). While, for inference time, KNN and RF spend the longest time, which all exceed $2 \mathrm{~s}$. KNN has a large number of calculations in the prediction stage. Therefore, inference takes more time. RF will produce multiple decision trees in model training, and it also depends on these decision trees for majority voting, resulting in a long time for model training and inference. For SVM, the model is complex, but its predictions only depend on the support vector, so the inference time is much shorter. In practical application, the inference time will have more influence on recognition efficiency, so Decision Tree and SVM seem to have more advantages, and since SVM is more accurate than Decision Tree, SVM was retained for further analysis. 


\subsection{Bistatic}

For bistatic radar, inspired by the work in [21], simulations on the radar robustness against varying aspect angles were conducted, and several different radar geometries are tested. From Fig. 3, it can be seen that the motion from monostatic radar becomes hard to distinguish when $\theta$ reaches $\pm 90^{\circ}$, and in bistatic radar, the range of aspect angles Doppler shifts are distinguishable is larger than for monostatic. For example, the spectrogram is clear for $\mathrm{CB} 10$ from $-90^{\circ}$ to 0 and $75^{\circ}$ to $90^{\circ}$; for $\mathrm{CB} 5$ from - $90^{\circ}$ to $30^{\circ}$; for $\mathrm{CB} 2.5$ from $-75^{\circ}$ to $60^{\circ}$.

It can be seen from Fig. 9. that the overall performance of CB2.5 is better than the other three schemes. Its accuracy is above $92 \%$ from $-75^{\circ}$ to $60^{\circ}$ and reached the highest value $(95 \%)$ at $30^{\circ}$. In comparison, the average accuracy of the monostatic radar is lower than that of $\mathrm{CB} 2.5$, and the accuracy begins to decline sharply when $\theta<-60^{\circ}$ and $\theta>60^{\circ}$, which means that the unaffected aspect angle range is lower than that of CB2.5.

It is also found in Fig. 10 that the classification performance parameters of CB2.5 are higher than those of other geometries when all the aspect angles are fused together to train one model for all angles. For example, the accuracy, sensitivity and specificities of $\mathrm{CB} 2.5$ have $2.2 \%, 7.7 \%$ and $1.5 \%$ improvement compared with monostatic radar. Therefore, it is inferred from the above results that the CB2.5 bistatic radar has stronger robustness against aspect angle change than monostatic radar.

\section{Conclusion}

Human activity recognition based on radar has been one of the research hotspots in recent years. It has great application value in the field of healthcare and security. In this paper, the classification of human activity in the monostatic radar environment is simulated first and then upgraded into a bistatic radar environment with configurable radar geometries to explore the improvement of robustness against changes in aspect angle. For feature extraction, 2D-PCA is applied, which can process images data more effectively compared with traditional PCA. It greatly reduces the data dimension, improving the efficiency of the algorithm. For classification, four machine learning algorithms are used, and it is concluded from the results that RF achieves the best results in classification accuracy, with a value up to $98.17 \%$, and it also has better stability than the other three models. The accuracy and stability of SVM are second only to RF, but it has a shorter inference time, which means the classification efficiency is higher for embedded processing.

This paper also establishes bistatic radar models to test the classification performance of different radar geometry at different aspect angles. The results show that CB2.5 is more advantageous than monostatic radar in accuracy under each aspect angle. It is also found in fused data classification that the classification performance of CB2.5 is better than monostatic radar, so these results indicate that CB2.5 has better aspect angle robustness.

This paper explored the robustness against varying aspect angles with bistatic radar and monostatic radar. Other geometries with MIMO [37] and multi-static geometries 
[21] with more transmitters and receivers could be explored to improve robustness. Another aspect of the simulation to be considered is the incorporation of channel propagation and reflection from the clutter in indoor environments to increase the realism of the simulations.

Furthermore, for the operation of several radars in indoor spaces, the study of radar signals with software-defined radar to avoid interference should be considered and their associated performance [38-41].

Last but not least, this simulator considered discrete activities, whereas, in reality, people perform actions in a continuum. So, the simulator should work to generate continuous data for further improvement of the techniques for automatic segmentation [4244].

\section{References}

1. J. L. Kernec et al., "Radar Signal Processing for Sensing in Assisted Living: The challenges associated with real-time implementation of emerging algorithms," IEEE Signal Processing Magazine, vol. 36, no. 4, pp. 29-41, 2019, doi: 10.1109/MSP.2019.2903715.

2. S. Z. Gurbuz and M. G. Amin, "Radar-Based Human-Motion Recognition With Deep Learning: Promising applications for indoor monitoring," IEEE Signal Processing Magazine, vol. 36, no. 4, pp. 16-28, 2019, doi: 10.1109/MSP.2018.2890128.

3. M. Abdur Rahman, M. M. Rashid, J. Le Kernec, B. Philippe, S. J. Barnes, F. Fioranelli, S. Yang, O. Romain, Q. H. Abbasi, G. Loukas, and M. Imran, "A Secure Occupational Therapy Framework for Monitoring Cancer Patients' Quality of Life," Sensors, vol. 19, no. 23, p. 5258, Nov. 2019.

4. H. Li, G. Cui, L. Kong, S. Guo, M. Wang and H. Yang, "Human Target Tracking for Small Aperture Through-wall Imaging Radar," 2019 IEEE Radar Conference (RadarConf), 2019, pp. 1-4.

5. E. Cippitelli, F. Fioranelli, E. Gambi and S. Spinsante, "Radar and RGB-Depth Sensors for Fall Detection: A Review," in IEEE Sensors Journal, vol. 17, no. 12, pp. 3585-3604, 15 June15, 2017, doi: 10.1109/JSEN.2017.2697077.

6. W. H. Widen, "Smart cameras and the right to privacy," Proc. IEEE, vol. 96, no. 10, pp. 1688-1697, 2008, doi: 10.1109/JPROC.2008.928764.

7. A. Shrestha, H. Li, J. Le Kernec and F. Fioranelli, "Continuous Human Activity Classification From FMCW Radar With Bi-LSTM Networks," in IEEE Sensors Journal, vol. 20, no. 22, pp. 13607-13619, 15 Nov.15, 2020.

8. X. Li, Z. Li, F. Fioranelli, S. Yang, O. Romain, and J. L. Kernec, "Hierarchical Radar Data Analysis for Activity and Personnel Recognition," Remote Sensing, vol. 12, no. 14, p. 2237, Jul. 2020.

9. A. Shrestha et al., "Cross-Frequency Classification of Indoor Activities with DNN Transfer Learning," 2019 IEEE Radar Conference (RadarConf), 2019, pp. 1-6, doi: 10.1109/RADAR.2019.8835844.

10. K. Rapoza. (2017) China's Aging Population Becoming More Of A Problem. Forbes. Available: https://www.forbes.com/sites/kenrapoza/2017/02/21/chinas-aging-population-becoming-more-of-a-problem/\#68537251140f.

11. A. Shrestha, J. Le Kernec, F. Fioranelli, Y. Lin, Q. He, J. Lorandel and O. Romain, Elderly care: activities of daily living classification with an $\mathrm{S}$ band radar. The Journal of Engineering, 2019: 7601-7606. 
12. V. C. Chen, The Micro-Doppler Effect in Radar. Artech House Publishers, 2011.

13. V C Chen, H Ling, Time-frequency transforms for radar imaging and signal analysis. 2002.

14. Imran, M.A., Ghannam, R., Abbasi, Q.H., Fioranelli, F. and Kernec, J.L. (2021). Contactless Radar Sensing for Health Monitoring. In Engineering and Technology for Healthcare (eds M.A. Imran, R. Ghannam and Q.H. Abbasi).

15. H. Li, A. Shrestha, H. Heidari, J. L. Kernec and F. Fioranelli, "A Multisensory Approach for Remote Health Monitoring of Older People," in IEEE Journal of Electromagnetics, RF and Microwaves in Medicine and Biology, vol. 2, no. 2, pp. 102-108, June 2018,

16. P. V. Klaine, M. A. Imran, O. Onireti, and R. D. Souza, "A survey of machine learning techniques applied to self-organizing cellular networks," Commun. Surveys Tuts., vol. 19, no. 4, pp. 2392-2431, 2017.

17. I. Goodfellow, Y. Bengio, and A. Courville, Deep Learning. Cambridge, MA: MIT Press, 2016.

18. B. Erol and M. G. Amin, "Radar Data Cube Processing for Human Activity Recognition Using Multisubspace Learning," vol. 55, no. 6, pp. 3617-3628, 2019.

19. R. G. Guendel, "Radar Classification of Contiguous Activities of Daily Living," Master Thesis, 2019, http://arxiv.org/abs/2001.01556.

20. Z. J. Baird, "Human Activity and Posture Classification Using Single Non-Contact Radar Sensor by Affairs in partial fulfillment of the requirements for the degree of Master of Applied Science," p. pp.55-87, 2017.

21. B. Zhou et al., "Simulation framework for activity recognition and benchmarking in different radar geometries," IET Radar, Sonar Navig., pp. 1-10, 2021, doi: 10.1049/rsn2.12049.

22. F. Fioranelli, M. Ritchie and H. Griffiths, "Bistatic human micro-Doppler signatures for classification of indoor activities," 2017 IEEE Radar Conference (RadarConf), 2017, pp. 0610-0615.

23. G. Manfredi, P. Russo, A. De Leo, and G. Cerri, "Efficient Simulation Tool to Characterize the Radar Cross Section of a Pedestrian in Near Field," Progress In Electromagnetics Research C, Vol. 100, 145-159, 2020. doi:10.2528/PIERC19112701

24. H. Du, Y. He and T. Jin, "Transfer Learning for Human Activities Classification Using Micro-Doppler Spectrograms," 2018 IEEE International Conference on Computational Electromagnetics (ICCEM), 2018, pp. 1-3.

25. H. Du, B. Ge, Y. Dai and T. Jin, "Knowing the Uncertainty in Human Behavior Classification via Variational Inference and Autoencoder," 2019 International Radar Conference (RADAR), 2019, pp. 1-4.

26. Y. Lin and J. Le Kernec, "Performance Analysis of Classification Algorithms for Activity Recognition Using Micro-Doppler Feature," 2017 13th International Conference on Computational Intelligence and Security (CIS), 2017, pp. 480-483.

27. Y. Lin, J. Le Kernec, S. Yang, F. Fioranelli, O. Romain and Z. Zhao, "Human Activity Classification With Radar: Optimization and Noise Robustness With Iterative Convolutional Neural Networks Followed With Random Forests," in IEEE Sensors Journal, vol. 18, no. 23, pp. 9669-9681, 1 Dec.1, 2018.

28. S. Vishwakarma, W. Li, C. Tang, K. Woodbridge, R. Adve, K. Chetty, "SimHumalator: An Open Source WiFi Based Passive Radar Human Simulator For Activity Recognition”, 2021, arXiv:2103.01677

29. Y. Kim and B. Toomajian, "Hand Gesture Recognition Using Micro-Doppler Signatures With Convolutional Neural Network," IEEE Access, vol. 4, pp. 7125-7130, 2016.

30. F. Fioranelli, M. Ritchie, and H. Griffiths, "Aspect angle dependence and multistatic data fusion for micro-Doppler classification of armed/unarmed personnel," IET Radar, Sonar \& Navigation, vol. 9, no. 9, pp. 1231-1239, 2015. 
31. B. Çă̆liyan and S. Z. Gürbüz, "Micro-Doppler-Based Human Activity Classification Using the Mote-Scale BumbleBee Radar," IEEE Geoscience and Remote Sensing Letters, vol. 12, no. 10, pp. 2135-2139, 2015.

32. R. Boulic, N. M. Thalmann, and D. Thalmann, "A global human walking model with realtime kinematic personification," Vis. Comput., vol. 6, no. 6, pp. 344-358, 1990.

33. M. Müller, T. Röder, M. Clausen, B. Eberhardt, B. Krüger, A. Weber, Documentation Mocap Database HDM05, Technical report, No. CG-2007-2, ISSN 1610-8892, Universität Bonn, June 2007.

34. J. W. Crispin and A. L. Maffett, "Radar cross-section estimation for simple shapes," Proceedings of the IEEE, vol. 53, no. 8, pp. 833-848, 1965.

35. [39] K. D. Trott, "Stationary Phase Derivation for RCS of an Ellipsoid," IEEE Antennas and Wireless Propagation Letters, vol. 6, pp. 240-243, 2007.

36. J. Yang, D. Zhang, A. F. Frangi, and J. Y. Yang, "Two-Dimensional PCA: A New Approach to Appearance-Based Face Representation and Recognition," IEEE Trans. Pattern Anal. Mach. Intell., vol. 26, no. 1, pp. 131-137, 2004.

37. F. Yang, F. Xu, F. Fioranelli, J. Le Kernec, S. Chang, and T. Long, Practical investigation of a MIMO radar system capabilities for small drones detection. IET Radar, Sonar and Navigation, 2021, Vol. 15, Issue 7 pp 760-774

38. J. Le Kernec, D. Gray and O. Romain, "Empirical analysis of chirp and multitones performances with a UWB software defined radar: Range, distance and Doppler," Proceedings of 2014 3rd Asia-Pacific Conference on Antennas and Propagation, 2014, pp. 1061-1064.

39. J. Le Kernec and O. Romain, "Empirical performance analysis of Linear Frequency Modulated pulse and Multitones on UWB software defined radar prototype," IET International Radar Conference 2013, 2013, pp. 1-6.

40. J. Le Kernec, "Inter-range-cell interference free compression algorithm: Performance in operational conditions," 2016 CIE International Conference on Radar (RADAR), 2016, pp. 15.

41. J. Le Kernec and O. Romain, "Performances of Multitones for Ultra-Wideband SoftwareDefined Radar," in IEEE Access, vol. 5, pp. 6570-6588, 2017.

42. H. Li, A. Mehul, J. Le Kernec, S. Z. Gurbuz and F. Fioranelli, "Sequential Human Gait Classification With Distributed Radar Sensor Fusion," in IEEE Sensors Journal, vol. 21, no. 6, pp. 7590-7603, 15 March15, 2021.

43. A. Shrestha, H. Li, J. Le Kernec and F. Fioranelli, "Continuous Human Activity Classification From FMCW Radar With Bi-LSTM Networks," in IEEE Sensors Journal, vol. 20, no. 22, pp. 13607-13619, 15 Nov.15, 2020.

44. H. Li, A. Shrestha, H. Heidari, J. Le Kernec and F. Fioranelli, "Bi-LSTM Network for Multimodal Continuous Human Activity Recognition and Fall Detection," in IEEE Sensors Journal, vol. 20, no. 3, pp. 1191-1201, 1 Feb.1, 2020. 